\section{Curricularización de la extensión universitaria para la promoción de la salud en la Universidad de La Habana. Un acercamiento a su conceptualización y praxis}

\author{
Amado Batista Mainegra \\ Profesor investigador del Centro de \\ Estudios para el Perfeccionamiento \\ de la Educación Superior. Universidad \\ de La Habana, Cuba. \\ amainegra@rect.uh.cu
}

\section{Odette González Aportela}

Directora de Extensión Universitaria, Universidad de La Habana.

odette@rect.uh.cu

\author{
Tania Ortiz Cárdenas \\ Directora del Centro de Estudios para \\ el Perfeccionamiento de la Educación \\ Superior, Universidad de La Habana. \\ tania@cepes.uh.cu
}

Integración de la docencia y la extensión /

Desafíos de gestión

RECEPCIÓN: 24/06/16

ACEPTACIÓN FINAL: 15/09/16

\section{Resumen}

A partir de las exigencias actuales de la educación superior cubana en la formación integral de los estudiantes y la participación activa y consciente de estos en dar solución a las demandas de la sociedad, la Universidad de La Habana incursiona desde el curso académico 2009-2010 en el desarrollo de la curricularización de la extensión universitaria. De ahí que el objetivo del presente trabajo sea socializar la experiencia de implementación de una estrategia metodológica de integración de procesos sustantivos como contribución a la conceptualización y praxis de la curricularización de la extensión universitaria en Cuba. La estrategia metodológica propuesta contribuye a la solución del diagnóstico inicial, caracterizado por la atomización de los procesos sustantivos universitarios y el estado deseado y necesario de integración de dichos procesos, específicamente en las acciones de promoción de salud, donde la extensión universitaria desempeña un rol dinamizador y sistémico y contribuye a la formación integral del joven profesional cubano.

\section{Palabras clave}

- Curricularización

- Extensión universitaria

- Estrategia metodológica

- Promoción de salud

\section{Resumo}

A partir das exigências atuais da educação superior cubana na formação global dos estudantes e participação ativa e consciente destes para dar solução às demandas da sociedade, a Universidade de La Habana começou a explorar a partir do ano letivo 2009-2010 no desenvolvimento da curricularização da extensão universitária. Assim, o objetivo deste trabalho é socializar a experiência de execução de uma estratégia de integração metodológica de integração de processos essenciais universitários como uma contribuição para a conceitualização e práxis da curricularização da extensão universitária em Cuba. A estratégia metodológica proposta contribui para a solução do diagnóstico inicial, caracterizado pela atomização dos processos essenciais universitários e o estado desejado e necessário de integração desses processos, especificamente nas ações de promoção da saúde, em que a extensão da universidade desempenha um papel dinamizador e sistémico e contribui para a formação integral do jovem profissional cubano.

Palavras-chave

- Curricularização

- Extensão universitária

- Estratégia metodológica

- Promoção da saúde
Para citación de este artículo

Batista Mainegra, A.; González Aportela, O. y Ortiz Cárdenas, T. (2016). Curricularización de la extensión universitaria para la promoción de la salud en la Universidad de La Habana. Un acercamiento a su conceptualización y praxis. En Revista $+E$ versión digital, (6), pp. 112-119. Santa Fe, Argentina: Ediciones UNL. 


\section{Introducción}

La razón de ser de la universidad contemporánea es satisfacer las necesidades sociales e individuales a partir de la preparación del ser humano como ente activo de la sociedad en la que se desempeña.

Para poder dar cumplimiento a esta misión, la universidad, en tanto institución formativa y transformadora, requiere aglutinar todo su quehacer y proyectarlo a través de un proceso fundamental: la extensión universitaria, facilitando el vínculo universidad-sociedad mediante la promoción de las diferentes formas de cultura (científico-tecnológica, artístico-literaria, cultura física, de salud, sociohumanística, económica, jurídica, político-ideológica, entre otras) para dar respuesta a las necesidades de superación y capacitación, por lo que contribuye al desarrollo cultural integral (Del Huerto, 2007; Pérez, 2010).

La extensión universitaria tiene la cualidad de ser integradora, pues se nutre de los contenidos de los demás procesos, aunque tiene personalidad propia y totalizadora. Esta cualidad de la extensión universitaria sustenta un nuevo paradigma para este procesofunción que descansa en un concepto amplio, diversificado con su consecuente aplicación en la práctica, asumiéndolo como un proceso formativo, dinámico, integrador y multifacético.

Siendo el ámbito educativo un escenario donde se favorecen procesos de aprendizaje, convivencia y crecimiento individual y social para el logro del bienestar integral de los estudiantes y su calidad de vida, es un lugar ideal para el desarrollo de acciones de promoción de la salud y de prevención de enfermedades, de amplio alcance y repercusión, ya que, como institución social, ejerce gran influencia en los estudiantes, de manera que sus familias y comunidad en general también se ven beneficiadas con dichas acciones.

Desde esta perspectiva se hace necesario potenciar actividades curriculares que tributen al desarrollo de una cultura de la salud, sustentada en los valores que deben estar presentes en el joven profesional cubano y contribuya de igual manera al desarrollo integral y saludable de los futuros profesionales, principalmente de aquellos cuyas esferas de actuación incluye al sector de la Salud Pública.

Es por ello que el objetivo del presente trabajo es socializar la implementación de una estrategia metodológica de integración de procesos sustantivos en la Universidad de La Habana como contribución a la conceptualización y praxis de la curricularización de la extensión universitaria en Cuba.

\section{Estado actual de la integración de procesos sustantivos universitarios para la promoción de salud: un diagnóstico necesario en la Universidad de La Habana}

Como parte de las tareas de investigación desarrolladas se realizó la caracterización de la integración de los procesos sustantivos universitarios en las acciones de promoción de salud en la Universidad de La Habana. Para ello se realizaron varias acciones investigativas que permitieron triangular la información y obtener una caracterización válida desde el punto de vista científico por medio de la determinación de las principales fortalezas y debilidades de este proceso, lo que constituyó el punto de partida para el diseño de la estrategia metodológica que se propone, de manera que ésta potenciara las fortalezas existentes para contrarrestar o eliminar las dificultades detectadas.

Al realizar la triangulación de todas las fuentes de información y los resultados de los instrumentos aplicados, se puede obtener una caracterización de la integración de procesos sustantivos universitarios en las acciones de promoción de salud que se desarrollan en la Universidad de La Habana, lo que conduce a plantear las fortalezas y debilidades de este proceso.

Se constatan como fortalezas:

- La voluntad política para el trabajo de promoción de salud, expresado en el funcionamiento de los Programas Nacionales que dirige la Dirección de Extensión Universitaria del Ministerio de Educación Superior, en correspondencia con el acuerdo 37/90 del 30 de octubre de 2000 del Comité Ejecutivo del Consejo de Ministros de Cuba, donde se adopta a la Promoción de la Salud como la estrategia del Estado cubano para desarrollar la salud del pueblo. - La voluntad de la dirección institucional, en especial, de la Dirección de Extensión Universitaria, por desarrollar un Programa Universitario de Promoción y Educación para la Salud, evidenciado en los resultados alcanzados y el nivel de compromiso de su equipo multidisciplinario de profesores.

- El funcionamiento de una Comisión Universitaria de Promoción y Educación para la Salud, que permite coordinar y asesorar en las facultades el trabajo de promoción de salud.

- La implementación de los Planes de Estudio “D”, que brindan la posibilidad de diseñar e impartir asignaturas electivas de promoción de salud como parte de lo que se define como curricularización de la extensión universitaria.

- La participación y coordinación nacional de la Universidad de La Habana en los Proyectos del Fondo Mundial de lucha contra el Sida, la tuberculosis y la malaria. 
- La impartición de un curso libre ${ }^{1}$ durante dos instancias sobre promoción de salud en el que se integran la formación de pregrado y la extensión universitaria.

- La existencia de un instrumento de investigación que se utiliza sistemáticamente para el diagnóstico de la comunidad estudiantil universitaria y la caracterización de los diferentes grupos de riesgo.

De la misma manera, se pudieron determinar las debilidades existentes, algunas de las cuales son causas originarias de otras, tal y como se muestra en el diagrama de causa-efecto o diagrama de Ishikawa que se presenta en la Figura 1, donde se pueden constatar las causas que contribuyen al origen y sostenimiento de la situación actual.

Tal situación apunta a la necesidad de diseñar e implementar, con el apoyo de las fortalezas determinadas anteriormente y para enfrentar las debilidades (disminuir o eliminar su efecto), una estrategia metodológica que propicie la integración de procesos sustantivos para la implementación de acciones de promoción de salud, donde la extensión universitaria juegue un papel dinamizador, sistémico e integrador de los demás procesos para el desarrollo de la promoción de salud, dado su carácter de procesofunción integradora y totalizadora.

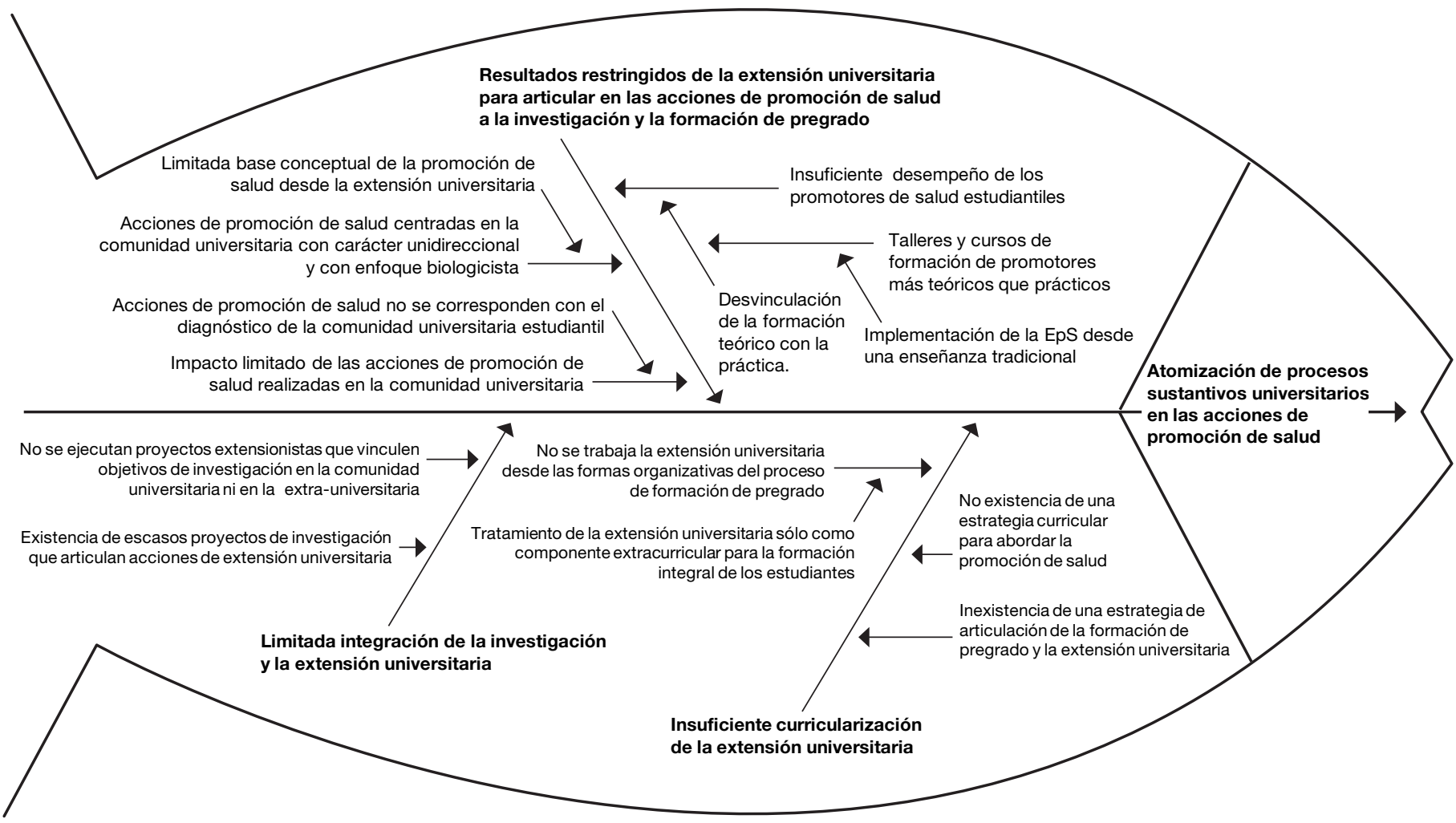

Fuente: Batista (2016)

1) Se define como curso libre o extensionista a toda aquella acción de formación, capacitación o superación, cuyo fin es la promoción de la cultura en su más amplia acepción, que es dirigida a estudiantes, trabajadores de la IES y la población en general, teniendo en cuenta las especificidades de estas po- blaciones y sus necesidades sentidas. También pueden ser conocidos, según la época del año en que se oferten, como cursos de verano (Batista, 2016). 
3. Estrategia metodológica de integración de procesos sustantivos universitarios: una propuesta en práctica para la curricularización de la extensión universitaria

El estudio de los fundamentos teórico-metodológicos de la extensión universitaria y de la promoción de salud permitió diseñar una estrategia metodológica de integración de procesos sustantivos universitarios que se caracterizara por ser abierta, flexible, contextualizada, transformadora, con un carácter sistémico y perspectivo. De esta forma se propició que el componente pedagógico incorporara al proceso de enseñanza-aprendizaje (PEA) prácticas de extensión universitaria con el apoyo o la utilización de elementos de la investigación científica (ver Figura 2). La estrategia metodológica diseñada responde a la definición que presenta Batista sobre este tipo particular de estrategia, al considerarla como:

"la secuencia de etapas (que incluyen actividades y acciones teórico-prácticas) que son planificadas, organizadas, ejecutadas y controladas por cualquier persona o grupo (profesor o colectivo docente), como vía más efectiva para alcanzar un estado necesario alcanzable, de acuerdo con objetivos concretos previamente delimitados que se articulan dialécticamente con la metodología propuesta en respuesta al diagnóstico, lo que permite que sean efectuados cambios y ajustes pertinentes acorde al contexto económico, político y socio-histórico, el contenido y los recursos disponibles (humanos, técnicos, materiales y financieros)". (2016:65)

\section{Figura 2}

Estrategia metodológica de integración de procesos sustantivos universitarios

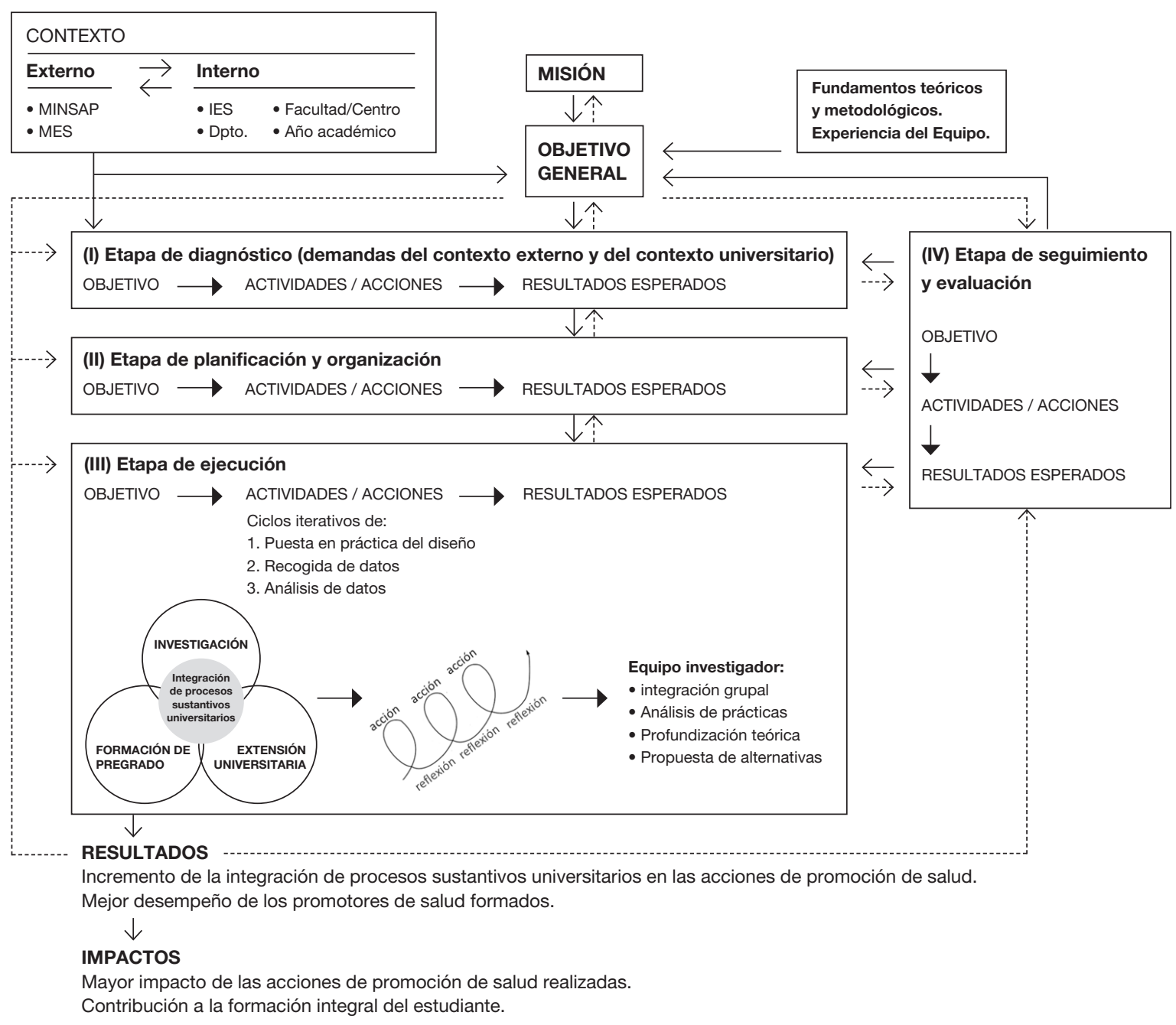

Fuente: Batista (2016) 


\section{6}

se hace necesario potenciar actividades

curriculares que tributen al desarrollo de

una cultura de la salud, sustentada en

los valores que deben estar presentes

en el joven profesional cubano

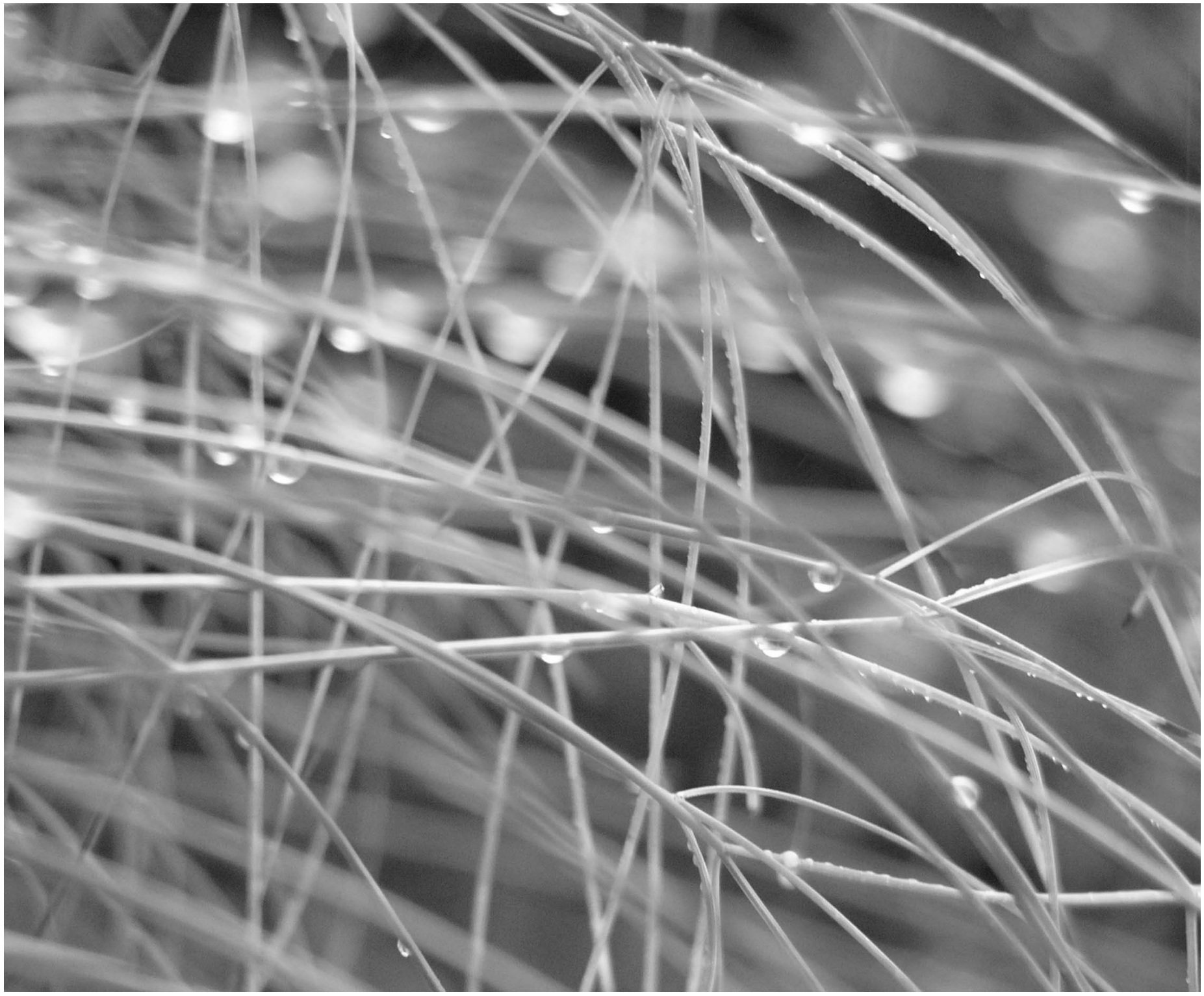

(c) Laura Malachesky 
A partir de esta definición, se concibe la estrategia metodológica propuesta como plan, pues indica el establecimiento de ciertos objetivos para la acción formulados de forma consciente, predeterminados y orientados a un fin de cambio, en coincidencia con el criterio de Deler (2007).

La estrategia metodológica tiene como objetivo general contribuir a la integración de procesos sustantivos universitarios en las acciones de promoción de salud que se desarrollen en la Universidad de La Habana, y para ello se sustenta en los siguientes presupuestos teóricos:

- El papel de la extensión universitaria como proceso integrador de los procesos sustantivos universitarios.

- El modelo educativo de la Educación para la Salud (EpS) como herramienta fundamental de la promoción de salud, con un abordaje de la salud de manera holística, con enfoque comunitario y participativo.

- El empleo de elementos del Enfoque Histórico-Cultural (EHC) como sustento teórico-metodológico de una enseñanza desarrolladora en las acciones de la EpS. Bajo este principio se aborda la EpS desde una perspectiva que no se limita únicamente a transmitir información sino que busca desarrollar conocimientos, habilidades y destrezas que contribuyan a la producción social de la salud, mediante procesos de aprendizajes dinámicos por los que se privilegia la comunicación de doble vía, así como la actitud crítica y participativa de los estudiantes, lo que no se lograría con una enseñanza tradicional.

- La utilización de la Investigación Basada en Diseño (IBD) como recurso metodológico en la puesta en práctica del diseño, recolección y análisis de datos, a partir de la implementación de ciclos iterativos en los que el equipo investigador, desde la integración grupal de los resultados, realice un análisis de la práctica y la profundización teórica necesaria que le permita proponer alternativas que enriquezcan la propia estrategia metodológica.

Para el caso particular de esta investigación, la integración de procesos sustantivos universitarios, de acuerdo con los fundamentos teórico-metodológicos explicitados, la estrategia se asume como un proceso. Una estrategia que parte de objetivos según el diagnóstico de necesidades, intereses y conocimientos, que está compuesta por elementos prácticos (actividades y acciones) en estrecha relación con la teoría. El propósito de esta estrategia contextualizada es transformar eficientemente la realidad en donde los recursos humanos representen el núcleo del desarrollo. De allí que se la considere desde una lógica sinérgica entre estado real y estado necesario, que transite por el cumplimiento de los objetivos (Deler, 2007).

En la concepción y desarrollo de la estrategia se asumió el modelo integral o educativo de la educación para la salud que está sustentado en el EHC. La concepción de enseñanza-aprendizaje sustentada en este enfoque es considerada como un proceso de socialización en que el estudiante se inserta como sujeto y objeto del aprendizaje y asume una posición activa y responsable en su proceso de formación de conocimientos, habilidades y valores, guiado por el profesor. Con este fundamento se abordó la educación para la salud desde una perspectiva que no se limita a transmitir información sino que busca desarrollar conocimientos, habilidades y destrezas que contribuyan a la producción social de la salud mediante procesos de aprendizajes dinámicos, en los cuales se privilegia la comunicación de doble vía así como la actitud crítica y participativa de los estudiantes, lo que no se lograría con una enseñanza tradicional.

La estrategia tiene como principales actores a los estudiantes universitarios y sus profesores, a los que se adicionan todos los miembros de la comunidad universitaria y otros actores de la comunidad extrauniversitaria, principalmente los que se seleccionen como público meta de las actividades extensionistas de promoción de salud.

La estrategia metodológica es capaz de expresar la forma específica de interrelación del objeto investigado con el entorno, por lo que, como sistema, puede ser comprendido en su conjunto de partes en constante interacción, no solo en su interior sino también con otros elementos imprescindibles del entorno universitario, interno y externo. Esto genera un esquema de análisis de la coherencia de cada una de las etapas de la estrategia metodológica, en la que el cumplimiento del objetivo general y de los objetivos de cada una de las etapas requiere la obtención de determinados resultados. Y para llegar a éstos es necesaria la ejecución de las actividades y acciones previstas.

Como resultado de la estrategia desarrollada se diseñaron y ejecutaron dos asignaturas electivas: "Promoción de Salud" y "Tuberculosis: una enfermedad re-emergente", ambas de carácter extensionista por definición, pues su finalidad común es propiciar la formación de una cultura de la salud en la comunidad universitaria. Las dos asignaturas diseñadas fueron impartidas a 257 estudiantes de las Licenciaturas en Microbiología, Biología, Bioquímica, Geografía y Contabilidad y Finanzas durante cinco cursos académicos, de 2010-2011 a 2014-2015, e hicieron factible la formación de los estudiantes como promotores de salud, los que realizaron un total de 65 acciones educativas a través de las cuales se han alcanzado 2582 personas, fundamentalmente adultos mayores y estudiantes y profesores miembros de la comunidad universitaria.

Desde esta perspectiva se asume la dimensión pedagógica de la extensión universitaria que propone Menéndez (2015) y que ha dado lugar a un proceso que en algunos países latinoamericanos se identifica como curricularización de la extensión universitaria (Tommasino y Rodríguez, 2010; Camilloni, 2013), proceso en el que se recupera y actualiza el debate acerca de la función social de la 
universidad. Según Camilloni:

"concebida como posibilidad y responsabilidad, a través de la articulación entre docencia, investigación y extensión (...) para seguir construyendo el vínculo universidad-sociedad y contribuyendo al diseño de nuevos espacios de aprendizaje para los estudiantes en el marco de las experiencias concretas de la extensión universitaria". (2013:8)

Por lo tanto, si bien la curricularización de la extensión universitaria es un término que comienza a abrirse paso en la educación superior cubana, no es algo nuevo en el escenario latinoamericano contemporáneo, en el que varias universidades han incorporado, desde el proceso extensionista, un grupo de actividades que tienden a integrar los procesos sustantivos universitarios y que se han definido como prácticas curriculares que articulan docencia, investigación y extensión en la formación de grado, que se vinculan con actores no universitarios en la búsqueda de contribuir a la resolución de sus problemáticas y que incorporan abordajes interdisciplinarios.

Teniendo en cuenta estas ideas, se asume en este trabajo a Batista, quien define, para el contexto cubano, a la curricularización de la extensión universitaria como:

"el proceso a través del cual, con el objetivo general de promover cultura, se diseñan e implementan, en el pregrado, asignaturas que incorporen naturalmente elementos de los tres procesos sustantivos universitarios, para incidir de manera más orgánica en el cumplimiento del encargo social de las Instituciones de Educación Superior y en la formación integral del futuro profesional”. (2016:24)

Esta definición se diferencia de sus similares de la región básicamente por su concepción, pues no se identifica con la práctica de instituir en el currículo universitario una asignatura sobre extensión universitaria ni el establecimiento de una carga horaria o de créditos para realizar actividades netamente extensionistas, elementos que caracterizan la concepción latinoamericana.

De esta manera, se hace un abordaje de la extensión universitaria que sustenta un nuevo paradigma para este proceso-función y que descansa en un concepto amplio, diversificado, con su consecuente aplicación en la práctica, como un proceso formativo, dinámico, integrador y multifacético que asume hoy su propia concepción: la preparación y consolidación de las instituciones de educación superior como organizaciones de cultura y la ampliación de la influencia recíproca e integración con la sociedad mediante la promoción de la cultura en el sentido más amplio.

Este proceso de curricularización de la extensión universitaria, desde la estrategia metodológica que se propone, contempla al estudiante como centro de un proceso de enseñanza-aprendizaje donde adquirirá conocimientos, habilidades y destrezas para el propio cuidado y para la implantación de estilos de vida saludables en el plano individual, para el logro del bienestar integral de la persona y su calidad de vida. Al mismo tiempo, las acciones de promoción de salud y de prevención de enfermedades que realice durante el desarrollo de las asignaturas electivas, o posteriormente, contribuirán a su crecimiento individual (se generan procesos de aprendizajes al enfrentarse a situaciones nuevas) y al desarrollo de una comunidad universitaria saludable.

De esta forma se desarrolla un proceso de formación y concienciación dirigido a despertar en los estudiantes el interés por la salud y la asunción de la responsabilidad individual y colectiva en su protección y fomento a través de la comprensión crítica del fenómeno salud/enfermedad, un proceso que permite facultar a las personas para que aumenten el control que tienen sobre su salud y para mejorarla.

Con la implementación de la estrategia no solo se garantiza una sólida formación académica, sino que se propicia un aprendizaje experiencial, según el criterio de Camilloni (2013).

Resultados similares fueron obtenidos por Rocerau y Vilanova (2011), lo que corrobora la importancia que tiene la incorporación de la extensión universitaria al currículo, evidenciada en el rol de formación que tiene la extensión universitaria para los estudiantes al ponerlos en contacto con la realidad. Algunos autores, como Camilloni (2011), han denominado "educación experiencial" a iniciativas como éstas.

De manera general se puede afirmar que la estrategia que se presenta, diseñada sobre la base de la integración de las funciones sustantivas o procesos principales de la universidad, contribuye a que éstos se orienten a cumplir la misión de la Universidad y así también se cumple, en parte, el encargo social de la misma, lo que hace de ésta una institución pertinente.

\section{Conclusiones}

El desarrollo de la estrategia planteada a partir de la integración de los procesos sustantivos universitarios con el desarrollo de prácticas integrales de extensión universitaria permitió contar con un espacio donde los alumnos realizaron actividades en las que, a través de la vinculación teoría-práctica, ponían a prueba en situaciones reales las habilidades y conocimientos teóricos que poseían, se autoevaluaban, enriquecían esos conocimientos y habilidades e identificaban nuevos problemas. Todo ello, además de contribuir a su formación integral, aporta a la educación para la ciudadanía y la formación ética de los estudiantes y facilita el conocimiento del contexto comunitario y social, al tiempo que les permite brindar un servicio de valor positivo en respuesta a demandas de satisfacción de necesidades exteriores o interiores a la Universidad. Sin lugar a dudas, estrategias como éstas deben formar parte de los modelos de formación de los profesionales que se desempeñarán en el campo de la Salud Pública. 


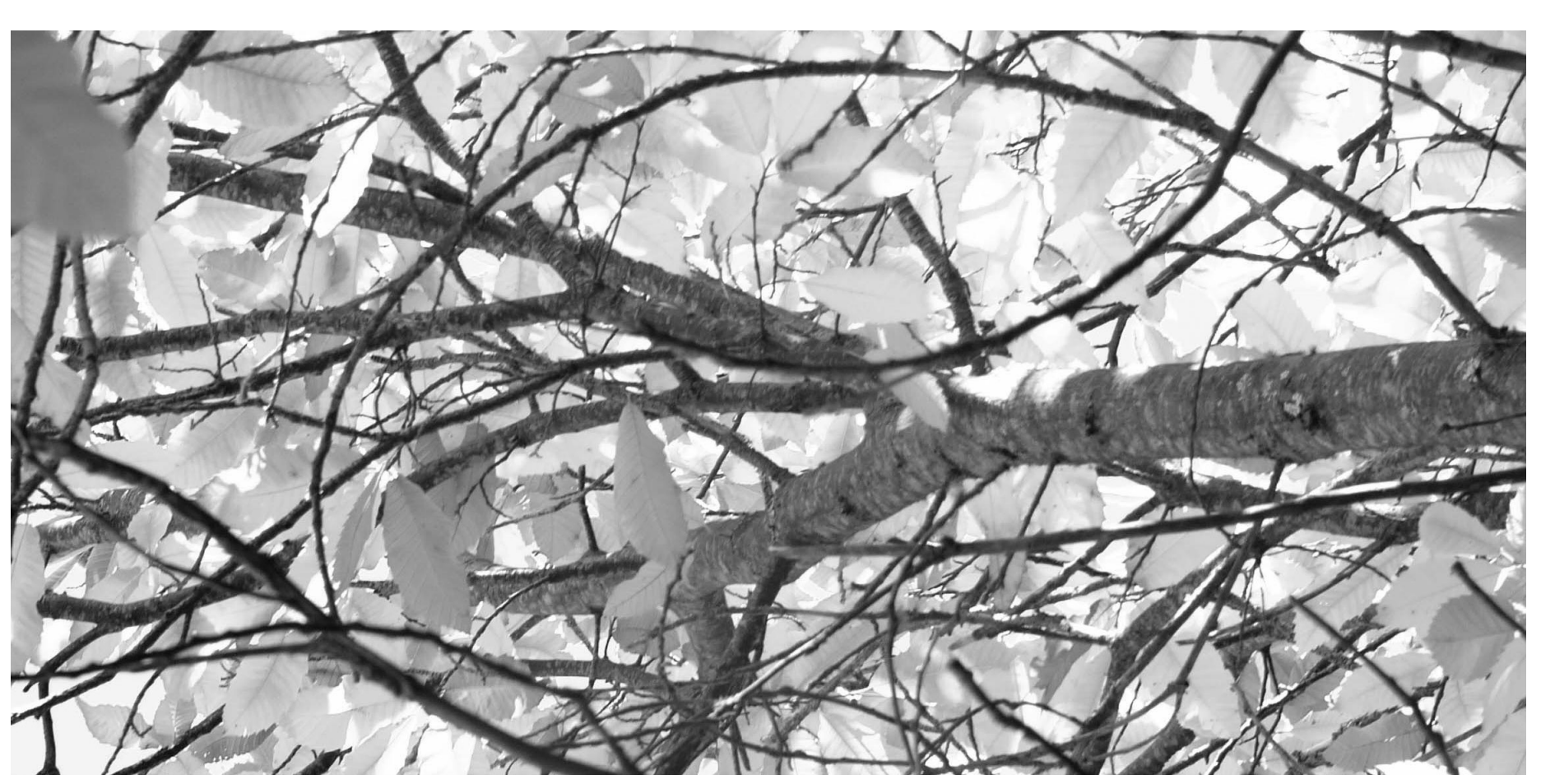

(C) Laura Malachesky

\section{Referencias bibliográficas}

Batista, A. (2016). Estrategia metodológica de integración de procesos sustantivos universitarios: contribución de la extensión universitaria a la promoción de salud en la Universidad de La Habana. Tesis para optar por el grado de Doctor en Ciencias de la Educación. Centro de Estudios para el Perfeccionamiento de la Educación Superior (CEPES), Universidad de La Habana.

Camilloni, A. (2011). "La inclusión de la extensión en la formación de los estudiantes de la Universidad Nacional del Litoral". En Revista +E, 1 (1), 76-79. Santa Fe: Ediciones UNL.

Camilloni, A. (2013). La inclusión de la educación experiencial en el currículo universitario". En Menéndez, G. y otros, Integración, docencia y extensión. Otra forma de enseñar y aprender. Santa Fe: Ediciones UNL.

Menéndez, G. y otros (2013). Integración docencia y extensión. Otra forma de enseñar y de aprender. Santa Fe: Ediciones UNL.

Deler, G. (2007). "La estrategia como resultado científico en la investigación pedagógica". Trabajo inédito.
Del Huerto, M. E. (2007). "La extensión universitaria como vía para fortalecer los vínculos Universidad-Sociedad desde la promoción de salud". Revista Cubana de Salud Pública, 33 (2), 10

Menéndez, G. (2015). "La extensión universitaria: aportes para la construcción de la red de observatorios y cátedras abiertas y/o libres". Recuperado de: http:// www.uleu.org/uleu_wp/ (29/05/2016)

Pérez, V. T. (2010). Conocimientos sobre el proceso de Extensión Universitaria en un área de salud". Revista Cubana de Medicina General Integral, 26 (1), 107-116. Rocerau, M. C. y Vilanova, S. (2011). Docencia y extensión: ampliando el espacio de la práctica. Libro de resúmenes del XI Congreso Iberoamericano de Extensión Universitaria. Memorias del Congreso. Santa Fe, Ediciones UNL.

Tommasino, H. y Rodríguez, N. (2010). Tres tesis básicas sobre extensión y prácticas integrales en la Universidad de la República. Cuadernos de Extensión nº 1. Integralidad: tensiones y perspectivas. Uruguay: UdelaR.

Curriculariza... I Batista Mainegra, A.; González Aportela, O. y Ortiz Cárdenas, T.| 119 